\title{
Anisotropy of zero-bias diffusive anomalies for different orientations of an external magnetic field
}

\author{
E.V. Sukhorukov, A.V. Khaetskii \\ Institute of Microelectronics Technology, Russian Academy of Sciences, 142432, Chernogolovka, \\ Moscow district, Russia
}

\begin{abstract}
We consider the influence of the electron-electron interaction on the nonlinearity of the current-voltage characteristic of the tunnel junction at low bias (diffusive anomaly) in the presence of the classical magnetic field. We present the theory of a new phenomenon which manifests itself in the strong anisotropy of a diffusive anomaly for different orientations of the magnetic field with respect to the interface of the tunnel junction. The nonlinear differential tunneling conductance has a universal magnetic field dependence, so that only the magnetic field component perpendicular to the interface is involved. In particular, when the magnetic field is parallel to the interface, the I-V characteristic does not depend on the value of the magnetic field.
\end{abstract}

PACS numbers: 73.40.Gk, 71.10.Pm

Typeset using REVTEX 


\section{INTRODUCTION.}

As it was shown by Altshuler and Aronov日, the electron-electron interaction strongly influences various physical properties of electron systems. Among those are conductivity, spin susceptibility and the one-particle density of states at the Fermi level. Suppression of the one-particle density of states at the Fermi level leads to appearance of small deviations from Ohm's law in the current-voltage characteristics of a tunnel junction at small voltages $V$ (zero-bias diffusive anomalies). These authors used the diagrammatic perturbative method. A more complete description of the nonlinearities in the I-V characteristics of tunnel junctions which allows to go beyond the perturbative treatment was given in Refs.(2 25). (Earlier the non-perturbative treatment of renormalization of one-particle density of states due to electron-electron correlations was given in Ref. (6). It was shown that the strength of the diffusive anomaly can be characterized by the effective frequency dependent impedance $(\omega \sim \mathrm{eV} / \hbar)$ which the electron feels when tunneling. Immediately after the electron tunnels, the system acquires an extra energy due to the interaction between this electron and the electrons of the liquid. Therefore, the electron density perturbation must spread in order to reach the final state. In this process the many-body system acquires some action which is proportional to the impedance mentioned above. The value of this effective impedance can be estimated as the resistance of the metal piece which the electron covers in time $\sim \hbar / \mathrm{eV}$ during its diffusion motion after the tunneling. The value of this impedance governs the magnitude of the deviations from Ohm's law. When the conductivity of the system is quite large, the effective impedance is much smaller than the quantum unit of resistance $h / e^{2}$ and, as a result, there is only a small deviation from the Ohmic behavior. In the present work we will assume that this condition is satisfied. The classical magnetic field influences the charge spreading (through the conductivity), hence, the zero-bias anomaly depends on the magnetic field value.

We present here the theory of a new phenomenon which manifests itself in the strong anisotropy of a diffusive anomaly for different orientations of the classical magnetic field 
with respect to the current direction. For definiteness, we considered the tunnel contact of two 3D semi-infinite samples and the magnetic field vector can have an arbitrary angle with the interface plane (see Fig. 11).

As is common, we consider the case of a metallic regime $\left(\epsilon_{F} \tau_{p} \gg 1, \tau_{p}\right.$ is the momentum relaxation time). We have solved the equations of classical electrodynamics which describe the propagation of the charges and electric fields for this geometry and obtained the following results. The nonlinear differential tunneling conductance has a universal magnetic field dependence, so that only the magnetic field component perpendicular to the interface is involved. This is related to the fact that the charge spreading after the tunneling has a completely different character for the classical magnetic field oriented perpendicular or parallel to the interface. Namely, the magnetic field perpendicular to the interface strongly reduces the transverse conductivity $\sigma_{x x}$ of the system (in the region of classically strong magnetic fields), hence, the spreading of the charge along the interface plane is strongly blocked. The effective impedance increases and has a universal dependence on the magnetic field $\left[1+\left(\omega_{c} \tau_{p}\right)^{2}\right]$ (multiplied by the impedance value for a zero magnetic field). This means that the nonlinear tunneling conductance also has this universal magnetic field dependence. For the magnetic field parallel to the interface the charge spreading along the interface plane is not blocked because this spreading easily occurs through the Hall drift along the interface. As a result, the I-V characteristic for this orientation of the magnetic field does not depend on the magnetic field value. This phenomenon resembles the absence of magnetoresistance in the metal at low temperatures for one group of carriers. In the case of arbitrary orientation only the perpendicular component of the magnetic field is involved because the contribution of the parallel component is canceled by the Hall electric fields.

The size effects in one-particle density of states in the presence of electron-electron correlations were studied in Ref. 77. This consideration was restricted to the case of a zero magnetic field. In contrast, the influence of the classical magnetic field on the electron density of states was studied only for the case of bulk metall. The authors of the latter did not take into account the important role of the surface of a metal in this phenomenon and could 
not obtain the strong dependence of the zero-bias anomaly on the orientation of an external magnetic field.

The results of present work explain the observations of the recent experiment 9 . In the latter the magnitude of the diffusive anomaly for the electron tunneling between two heavily doped GaAs samples was measured for two different orientations of the external magnetic field parallel and perpendicular to the interface. It was observed that the magnitude of the diffusive anomaly does not depend on the magnetic field value in the case when the magnetic field is parallel to the interface and strongly depends on it in the case of perpendicular orientation.

\section{FORMALISM.}

Let us consider the concrete geometry of the experiment. We assume that the leads are two 3D semi-infinite conducting samples isolated by a thin high barrier of width $d$ (see Fig. 四). If a negative voltage is applied to the left lead, the electron may tunnel through the barrier from the left lead to the right one leaving a hole behind. The magnetic field applied governs the charge spreading process after tunneling. In general, the magnetic field is arbitrary oriented so that the diffusion coefficients $D_{k m}$ depend not only on the magnetic field value, but also on the angle $\theta$ between the magnetic field and the plane of the insulating layer.

We use the tunnel Hamiltonian method. The total Hamiltonian of the problem consists of the following parts:

$$
\hat{H}=\hat{H}_{R}+\hat{H}_{L}+\hat{H}_{i m p}+\hat{H}_{T}
$$

Here $\hat{H}_{R, L}$ describes the electron system in the right and the left leads with the electronelectron interaction taken into account. The Hamiltonian $\hat{H}_{i m p}$ describes the electron scat-

tering by the impurities and the tunnel term $\hat{H}_{T}=\hat{A}+\hat{A}^{\dagger}$ describes the transitions of electrons through the insulating layer, 


$$
\hat{A}=\int_{\substack{x>d / 2 \\ x^{\prime}<-d / 2}} d^{3} \mathbf{r} d^{3} \mathbf{r}^{\prime} T\left(\mathbf{r}, \mathbf{r}^{\prime}\right) \psi^{\dagger}(\mathbf{r}) \psi\left(\mathbf{r}^{\prime}\right) .
$$

The corresponding expression for the current operator is

$$
\hat{I}=i\left(\hat{A}-\hat{A}^{\dagger}\right)
$$

As usual, the current is calculated in the second-order perturbation theory in the tunnel Hamiltonian. For arbitrary voltage $V$ the current can be obtained 10 by the analytical continuation of the following Matsubara Green's function determined at $\omega_{n}=2 \pi T n$,

$$
I(V)=2 e \operatorname{Im}\left\{\left.\int_{0}^{\beta} d \tau e^{i \omega_{n} \tau}\left\langle\hat{T} \hat{A}(\tau) \hat{A}^{\dagger}(0)\right\rangle\right|_{i \omega_{n} \rightarrow-e V+i 0}\right\} .
$$

From now on we assume that $e>0$ and $\hbar=1$. This Green's function is calculated in the standard fashion by averaging over the electrodynamic field configurations $\mathbf{A}^{\mathrm{S}}$ and can be expressed in terms of the effective action which describes the low-frequency electrodynamic modes of the system excited during the tunneling. When the action is small $(S(\tau) \ll 1)$, one can get the following simple expression for the small correction to the current-voltage characteristic at $T=0$ :

$$
R_{t} \frac{\partial^{2} I}{\partial V^{2}}=\frac{2 e}{\pi} J m\left\{\left.S(\omega)\right|_{i \omega \rightarrow-e V+i 0}\right\}
$$

where $R_{t}$ is the tunnel resistance of the junction.

At low frequencies ( $\omega \tau_{p} \ll 1, \tau_{p}$ is the momentum relaxation time), when the diffusion approximation is valid, one can get the following formula for the action $\mathrm{B}$ :

$$
S(\omega)=\frac{1}{2} \int d^{3} \mathbf{r} \rho_{-\omega}(\mathbf{r}) \phi_{\omega}(\mathbf{r}),
$$

where $\phi_{\omega}(\mathbf{r})$ is the electrodynamical potential excited by the tunneling electron, and $\rho_{\omega}(\mathbf{r})=$ $\int_{-\infty}^{+\infty} d \tau \exp (i \omega \tau) \rho(\mathbf{r}, \tau)$ is the Fourier transformation of the probability density to find the electron (hole) at point $\mathbf{r}$ if $\mathbf{r}_{0}$ is the point of tunneling on the interface of the tunnel junction. This quantity is just a common electron-hole (diffusion) propagator which stands 
in usual diagrammatic approach 1 . $\rho_{\omega}(\mathbf{r})$ obeys the diffusion equation in imaginary time in the presence of the magnetic field (different signs correspond to different leads),

$$
|\omega| \rho_{\omega}(\mathbf{r})-\sum_{k, m=x, y, z} D_{k m} \nabla_{k} \nabla_{m} \rho_{\omega}(\mathbf{r})=\mp e \operatorname{sign}(\omega) \delta\left(\mathbf{r}-\mathbf{r}_{0}\right)
$$

with the boundary conditions that there is no diffusive current across the surfaces of the leads:

$$
\left.\sum_{k} D_{x k} \nabla_{k} \rho_{\omega}(\mathbf{r})\right|_{x= \pm d / 2}=0
$$

This boundary condition is imposed at the distance from the surface which is larger than mean free path. It should be noted that in the case of $\omega_{c} \tau_{p} \gg 1$ the diffusion coefficients are renormalized due to the existance of skipping orbits at the distance of the order of cyclotron radius $R_{c}$ from the surface. However, in the diffusion approximation we consider here the contribution of the sliding current to the action $S(\omega)$ is small with respect to the parameter $q R_{c} \ll 1$, where $q^{-1}$ is the characteristic length scale in the problem.11

In the coordinate system when the magnetic field is parallel to the $z$-axis, the diffusion coefficients have the following form ( $\omega_{c}$ is the cyclotron frequency):

$$
\begin{gathered}
\tilde{D}_{z z}=D_{0}=\frac{v_{F}^{2} \tau_{p}}{3}, \quad \tilde{D}_{x x}=\tilde{D}_{y y}=\frac{D_{0}}{1+\omega_{c}^{2} \tau_{p}^{2}}, \\
\tilde{D}_{x y}=-\tilde{D}_{y x}=-\operatorname{sign}(\omega) D_{0} \frac{\omega_{c} \tau_{p}}{1+\omega_{c}^{2} \tau_{p}^{2}} .
\end{gathered}
$$

For an arbitrary orientation of the magnetic field in the $\mathrm{x}-\mathrm{z}$ plane the diffusion coefficients can be obtained from the diffusion matrix $\tilde{D}$ (Eqs. (9, 10) ) by the rotation over the angle $\theta$ around the $y$-axis: $D(\theta)=\hat{U}^{T}(\theta) \tilde{D} \hat{U}(\theta)$.

We assume that the conductivity $\sigma$ of the leads is large, so for the frequencies in question ( $\omega \ll \sigma)$ we use the electroneutrality condition (Debye radius $r_{D}=0$ ) when calculating the electrodynamical potential $\phi_{\omega}(\mathbf{r})$. In this case there are no charges in the 3D leads except the $2 \mathrm{D}$ charges $\pm Q_{\omega}\left(\mathbf{r}_{\|}\right)$on their surfaces, and $\phi_{\omega}(\mathbf{r})$ obeys the system of the following equations in imaginary time: 


$$
\begin{gathered}
\sum_{k} \nabla_{k} j_{k}(\omega, \mathbf{r})=0 \\
j_{k}(\omega, \mathbf{r})=-\sum_{m} \sigma_{k m} \nabla_{m} \phi_{\omega}(\mathbf{r}) \\
\left.j_{x}\left(\omega, \mathbf{r}_{\|}\right)\right|_{x= \pm d / 2}=-|\omega| Q_{\omega}\left(\mathbf{r}_{\|}\right)-e \operatorname{sign}(\omega) \delta\left(\mathbf{r}_{\|}\right)
\end{gathered}
$$

Conductivity is related to the diffusion tensor through the Einstein relation, $\sigma_{k m}=$ $e^{2} \nu_{F} D_{k m}$, where $\nu_{F}$ is the density of states for noninteracting electrons at the Fermi surface. Outside the leads $\phi_{\omega}(\mathbf{r})$ obeys the Laplace equation. Since for the small frequencies considered here the characteristic spatial scale of the variation of $\phi_{\omega}$ inside the leads is much larger than $d$, we can neglect the electric fields in the leads while finding the relation between values of $\phi_{\omega}$ and $Q_{\omega}$ at the surfaces. Hence, we obtain a simple relation

$$
\left.\phi_{\omega}\left(\mathbf{r}_{\|}\right)\right|_{x= \pm d / 2}= \pm 2 \pi d Q_{\omega}\left(\mathbf{r}_{\|}\right) / \epsilon_{i}
$$

where $\epsilon_{i}$ is the dielectric constant of the insulating layer.

It should be noted that according to Refs.( 2 (4) the value $Z(\omega)=e^{-2} \omega S(\omega)$ can be thought as the effective frequency dependent impedance which the electron feels when tunneling.

Finally, we would like to mention the connection between the method used in the present paper and the diagrammatic approach 1 . Calculation of the correlator in Eq.(4) corresponds (in the lowest order) to the account of diagrams a) and b) in Fig. 2. Diagram a) is the exchange correction to one-particle density of states in each lead. Diagram b) contributes to the correlator due to excitation of the gapless interface plasmon during electron tunneling. We do not take into account Hartree diagram c) which is small in parameter $\lambda_{F} / r_{D} \ll 1$ because we consider the case of the weakly non-ideal electron gas.

\section{CALCULATION OF ACtion $S(\omega)$.}

Differential Eq. (7) with boundary condition (8) can be easily solved by the Fourier transformation with respect to the $y$ and $z$ coordinates along the surface plane. Then, for 
the charge density in the right lead $(x>d / 2)$ we obtain

$$
\rho_{\omega}(x, \mathbf{q})=-\frac{e \operatorname{sign}(\omega)}{\Delta D_{x x}-i q_{y} D_{x y}} \exp \left[-\left(i q_{z} D_{x x}^{-1} D_{x z}+\Delta\right)(x-d / 2)\right],
$$

where $\Delta^{2}=D_{x x}^{-1}\left[|\omega|+D_{y y} q_{y}^{2}+\left(D_{z z}-D_{x x}^{-1} D_{x z}^{2}\right) q_{z}^{2}\right]$. A similar procedure with Eqs. (11-14) for the electrodynamic potential $\phi_{\omega}$ gives

$$
\phi_{\omega}(x, \mathbf{q})=-\frac{e \operatorname{sign}(\omega)}{(2 \pi d)^{-1} \epsilon_{i}|\omega|+\delta \sigma_{x x}-i q_{y} \sigma_{x y}} \exp \left[-\left(i q_{z} \sigma_{x x}^{-1} \sigma_{x z}+\delta\right)(x-d / 2)\right]
$$

with $\delta^{2}=\sigma_{x x}^{-1}\left[\sigma_{y y} q_{y}^{2}+\left(\sigma_{z z}-\sigma_{x x}^{-1} \sigma_{x z}^{2}\right) q_{z}^{2}\right]$. Then, for the characteristic frequency scale of the problem $\left(\omega \sim D q^{2}, q \ll l_{i}^{-1}\right.$ and $\left.l_{i}=v_{F} \tau_{p}\right)$ the term $(2 \pi d)^{-1} \epsilon_{i}|\omega|$ is small compared to others in the denominator of the right hand side of Eq. (16), provided $d \gg r_{D}^{2} / l_{i}\left(r_{D}^{2}=\right.$ $\left.D_{0} / \sigma_{0}\right)$. The last inequality is usually satisfied for tunnel junctions, and we omit this term in the expression for $\phi_{\omega}$ in further calculations. This approximation actually means that the spreading of the charges in the left and right leads occurs absolutely independently. It is determined by the diffusive modes and is not effected by surface plasmon modes which are not excited in the frequency range considered (it means that we neglect the contribution of diagram b) in Fig. 2).

By inversion of the coordinates and the sign of the electron charge in Eqs. (15, 16) we can obtain the potential and the charge density in the left lead. Then, $S(\omega)$ in Eq. (6) is given by the integral over the right lead multiplied by 2. Carrying out the integration over $x$ and over $\mathbf{q}$, we present $S(\omega)$ in the following universal form:

$$
S(\omega)=S_{0}(\omega) F(H, \theta), \quad S_{0}(\omega)=-\frac{e^{2}}{2 \pi \sigma_{0} \sqrt{|\omega| D_{0}}}, \quad F(H, \theta)=1+\omega_{c}^{2} \tau_{p}^{2} \sin ^{2} \theta .
$$

\section{NONLINEAR DIFFERENTIAL CONDUCTANCE.}

In the expression for $S(\omega)$ (see Eq. (17)) the dependence on $\omega$ is extracted in factor $S_{0}(\omega)$, i.e. the action for $H=0$. Factor $F(H, \theta)$ depends only on the magnetic field. Hence, to obtain the correction to the current-voltage characteristic we can easily perform the analytical continuation in Eq. (17), 


$$
R_{t} \frac{\partial^{2} I}{\partial V^{2}}=\frac{e^{3}}{\pi^{2} \sigma_{0} \sqrt{2 e V D_{0}}} F(H, \theta)
$$

It seems to be more convenient to represent this result in terms of the correction $\delta R(H, \theta)$ to the Ohmic resistance of the tunnel junction. To do this, we integrate the above equation over $V$,

$$
R_{t} \frac{\partial I}{\partial V}=\left[\frac{e^{2}}{\pi^{2} \sigma_{0}}\left(\frac{2 e V}{D_{0}}\right)^{1 / 2}-\frac{\delta R(0)}{R_{t}}\right] F(H, \theta),
$$

so that the correction to the resistance at zero bias is $\delta R(H, \theta)=\delta R(0) F(H, \theta)$, and $\delta R(0)$ can be estimated by comparison of the two terms in Eq. (19) at $\mathrm{eV} \sim 1 / \tau_{p}: \delta R(0) / R_{t} \sim$ $\left(\lambda_{F} / l_{i}\right)^{2}$.

Function $F(H, \theta)$ governs the dependence of the zero-bias anomaly on the magnetic field. As it follows from Eq. (17), there is a strong anisotropy of the diffusive anomaly with respect to the orientation of the magnetic field. In particular, if the magnetic field is exactly parallel to the interface, then $F(H, 0)=1$ and the correction to the resistance of the tunnel junction does not depend on the magnetic field value. In our opinion, this quite interesting effect has the same physical origin as the absence of the magnetoresistance in the metal for one group of carriers. Namely, additional spreading of the charge along the interface plane due to the Hall electric fields that appear in this geometry cancels exactly the blocking of the charge spreading due to decreasing $\sigma_{x x}$ in the magnetic field. In contrast, for the case when the magnetic field is perpendicular to the interface plane, the Hall electric fields do not occur and the charge spreading is governed only by $\sigma_{x x}$. As a result, for $\theta=\pi / 2$ we have $F(H, \pi / 2)=1+\omega_{c}^{2} \tau_{p}^{2}$, which is just the magnetic field dependence of $\sigma_{x x}^{-1}$. It is worth mentioning that in general factor $F(H, \theta)$ depends only on the component of the magnetic field $H_{x}$ perpendicular to the interface.

In conclusion, we have considered the influence of the classical magnetic field on the diffusive anomaly in the I-V characteristic of the tunnel junction. We have shown that the nonlinear differential tunneling conductance strongly depends on the orientation of the magnetic field with respect to the interface plane. This phenomenon is explained by the different 
character of charge spreading after tunneling for different orientations of the magnetic field. The result of this work explains the observation of the recent experiment 9 .

This work was financially supported by RFFI Grant No. 96-02-17757. E.V.S. wishes to thank prof. K. Ishikawa for the kind hospitality during his visit to the Department of Physics, Hokkaido University, Sapporo, Japan, where part of the work was done. A.V.Kh. thanks Dr. Yu. Nazarov for useful discussion of the results of this work. 


\section{REFERENCES}

${ }^{1}$ B.L. Altshuler, and A.G. Aronov, in Electron-Electron Interaction in Disordered Systems, edited by A.L. Efros, and M. Pollak (Elsevier, Amsterdam, 1985).

${ }^{2}$ G.-L. Ingold, and Yu.V. Nazarov, in Single Charge Tunneling, edited by H. Grabert and M. H. Devoret (Plenum, New York, 1992).

${ }^{3}$ Yu.V. Nazarov, Zh. Eksp. Teor. Fiz. 95, 975 (1989) [Sov. Phys. JETP 68, 561 (1989)].

${ }^{4}$ Yu.V. Nazarov, Fiz. Tverd. Tela 31, 188 (1989) [Sov. Phys. Solid State 31, 1581 (1989)].

${ }^{5}$ L.S. Levitov, and A.V. Shytov, unpublished (1995).

${ }^{6}$ A.M. Finkelstein, Zh. Eksp. Teor. Fiz. 84, 168 (1983); [Sov. Phys. JETP 57, 97 (1983)].

${ }^{7}$ B.L. Altshuler, A.G. Aronov, and A.Yu. Zuzin, Zh. Eksp. Teor. Fiz. 86, 709 (1984); [Sov. Phys. JETP 59, 415 (1984)].

${ }^{8}$ B.L. Altshuler, and A.G. Aronov, Zh. Eksp. Teor. Fiz. 77, 2028 (1979) [Sov. Phys. JETP 50, $968(1979)]$.

${ }^{9}$ Yu.V. Dubrovskii, Yu.N. Khanin, T.G. Andersson, U. Gennser, D.K. Maude, and J.-C. Portal, Zh. Eksp. Teor. Fiz. 109, 868 (1996); [Sov. Phys. JETP 82, 467 (1996)].

${ }^{10}$ G.D. Mahan, Many-Particle Physics (Plenum, New York, 1981).

${ }^{11}$ In $2 \mathrm{D}$ case with taking into account Landau quantization the presence of skipping orbits near the boundary may be important for the formulation of the boundary conditions for the diffusion propagator [D.L. Maslov and Daniel Loss, Phys. Rev. Lett. 71, 4222 (1993)]. 


\section{FIGURES}

FIG. 1. The tunnel geometry. The magnetic field vector constitutes angle $\theta$ with the interface plane.

FIG. 2. Diagrams which contribute in the lowest order to two-particle Green's function Eq.(4) are shown. Diagrams a) and c) describe correspondingly exchange and Hartree corrections to one-particle Green's function in each lead. Diagram b) describes the interaction of electrons in different leads. Wavy line is dynamically screened Coulomb interaction. Diffusion vertexes are shown by shaded regions. 